\title{
Geotecnologia e mudanças climáticas em vegetação de altitude
}

\author{
Geotechnology and climate change in altitude vegetation \\ Geotecnología y cambios climáticos en la vegetación de altitud
}

Recebido: 28/06/2021 | Revisado: 05/07/2021 | Aceito: 09/07/2021 | Publicado: 18/07/2021

\author{
Dhonatan Diego Pessi \\ ORCID: https://orcid.org/0000-0003-0781-785X \\ Universidade Federal de Mato Grosso do Sul, Brasil \\ E-mail: dhonatan.pessi@gmail.com \\ Marco Antonio Diodato \\ ORCID: https://orcid.org/0000-0002-9088-836X \\ Universidade Federal Rural do Semi Árido, Brasil \\ E-mail: diodato@ufersa.edu.br \\ Normandes Matos da Silva \\ ORCID: https://orcid.org/0000-0002-4631-9725 \\ Universidade Federal de Rondonópolis, Brasil \\ E-mail: normandes@ufr.edu.br \\ Alfredo Marcelo Grigio \\ ORCID: https://orcid.org/0000-0002-2094-9710 \\ Universidade do Estado do Rio Grande do Norte, Brasil \\ E-mail: alfredogrigio1970@gmail.com \\ Camila Leonardo Mioto \\ ORCID: https://orcid.org/0000-0002-6951-9527 \\ Universidade Federal de Rondonópolis, Brasil \\ E-mail: ea.mioto@gmail.com \\ Vinícius de Oliveira Ribeiro \\ ORCID: https://orcid.org/0000-0002-4373-1132 \\ Universidade Estadual de Mato Grosso do Sul, Brasil \\ E-mail: viniciusoribeiro@yahoo.com.br \\ Antonio Conceição Paranhos Filho \\ ORCID: https://orcid.org/0000-0002-9838-5337 \\ Universidade Federal de Mato Grosso do Sul, Brasil \\ E-mail: antonio.paranhos@ufms.br
}

\section{Resumo}

A análise bibliométrica é considerada um estudo rápido e eficiente para avaliar as tendências de pesquisa e as redes acadêmicas em diferentes disciplinas de pesquisa. O objetivo desse estudo foi realizar uma análise bibliométrica e apresentar uma visão geral das tendências de pesquisa sobre a variabilidade espacial e temporal da vegetação de altitude em resposta às mudanças climáticas com o auxílio de geotecnologias. Para tanto, foi realizada a pesquisa bibliográfica usando o banco de dados Web of Science em 11 de dezembro de 2020, para reunir publicações acadêmicas sobre alterações espaço-temporais de vegetação de altitude em resposta às mudanças climáticas, entre os anos de 1945 e 2020. Obteve-se um total de 54 publicações com resumos alinhados. Foi realizada a leitura de todos esses documentos e chegou-se ao número de 27 publicações que atenderam a todos os critérios relacionados ao objetivo dessa análise. A análise demonstrou que o número de publicações relacionadas ao tema desta pesquisa ainda é muito pequeno e restrito a poucos países e a tipos de vegetação específicas de determinadas áreas alpinas.

Palavras-chave: Vegetação campina; Publicações científicas; Mudanças climáticas; Variações espaço-temporais; Altitude; Distribuição da vegetação; Geotecnologias.

\begin{abstract}
Bibliometric analysis is considered a quick and efficient study to assess research trends and academic networks in different research disciplines. The aim of this study was to perform a bibliometric analysis and present an overview of research trends on the spatial and temporal variability of highland vegetation in response to climate change with the aid of geotechnologies. To this end, a bibliographic search was carried out using the Web of Science database on December 11,2020 , to gather academic publications on spatiotemporal changes in altitude vegetation in response to climate change, between the years of 1945 and 2020. A total of 54 publications with aligned abstracts were obtained. All these documents were read and the number of 27 publications that met all the criteria related to the objective of this analysis was reached. The analysis showed that the number of publications related to the topic of this research is still very small and restricted to a few countries and specific vegetation types in certain alpine areas.
\end{abstract}

Keywords: Grasslands; Scientific publications; Climate change; Spatial-temporal variations; Altitude; Vegetation distribution; Geotechnologies. 


\section{Resumen}

El análisis bibliométrico se considera un estudio rápido y eficiente para evaluar las tendencias de investigación y las redes académicas en diferentes disciplinas de investigación. El objetivo de este estudio fue realizar una análisis bibliométrica y presentar una visión general de las tendencias de investigación sobre la variabilidad espacial y temporal de la vegetación de altitud en respuesta al cambio climático con la ayuda de geotecnologías. Para ello, se realizó una búsqueda bibliográfica utilizando la base de datos de Web of Science el 11 de diciembre de 2020, para recopilar publicaciones académicas sobre cambios espacio-temporales en la vegetación de altitud en respuesta al cambio climático, entre los años de 1945 y 2020. Obtuvieron un total de 54 publicaciones con resúmenes alineados. Se leyeron todos estos documentos y se alcanzó el número de 27 publicaciones que cumplieron con todos los criterios relacionados con el objetivo de este análisis. El análisis mostró que el número de publicaciones relacionadas con el tema de esta investigación es aún muy pequeño y restringido a unos pocos países y tipos de vegetación específicos en ciertas áreas alpinas.

Palabras clave: Vegetación de pradera; Publicaciones cientificas; Cambios climáticos; Variaciones espaciotemporales; Altitud; Distribución de la vegetación; Geotecnologías

\section{Introdução}

Nas últimas décadas tem se discutido ativamente sobre as mudanças climáticas atuais, por ser um processo complexo, que tem como resultado a alteração da química da atmosfera e do equilíbrio global de energia, resultando em temperaturas elevadas, padrões de precipitação alterados e eventos climáticos extremos (Correa-Díaz et al., 2020; Blunden \& Arndt, 2018).

Neste contexto, o aquecimento global traz muitos desafios para o desenvolvimento da sociedade humana, e também para o ecossistema terrestre, pois o aquecimento global tem causado distúrbios florestais (Ma et al., 2019).

A frequência e gravidade dos distúrbios aumentarão sob a influência das mudanças climáticas nos ecossistemas florestais, devido ao crescimento e distribuição de plantas dependentes das condições climáticas e de seu habitat, pois a distribuição da vegetação demonstrou ser muito sensível ao clima (Ma et al., 2019; Wang et al., 2014)

Dado que a vegetação de alta elevação, como por exemplo os Campos de Altitude, são um tipo de ecossistema sensível ao clima, fazendo deste tipo de vegetação um bioindicador adequado para monitorar os efeitos das mudanças climáticas, uma vez que experimentam mudanças mais rápidas de temperatura do que os locais de baixa elevação (Correa-Díaz et al., 2020; Pepin et al., 2015). Espera-se que a vegetação que cresce na linha das árvores (vegetação mais densa), modulem o processo de formação de madeira de acordo com as imposições climáticas, garantindo o transporte de água e suporte físico enquanto equilibra as trocas para transpiração e fotossíntese (Correa-Díaz et al., 2020), sendo que variações nos padrões de distribuição de água e temperatura é um fator chave na distribuição espacial das árvores (Tao et al., 2018).

A alteração na distribuição espacial e temporal da vegetação é um importante indicador das respostas dinâmicas dos ecossistemas às mudanças climáticas (Zhang et al., 2019a; Jeong et al., 2011), portanto, pesquisas sobre a distribuição espaçotemporal da vegetação não só pode melhorar nossa compreensão das respostas da vegetação quanto às mudanças climáticas, mas também tem um significado importante para aumentar a precisão das simulações de troca de matéria-energia entre o clima e a vegetação (Pouliot et al., 2009; Zhang et al., 2019a).

Por conta da importância desse tema que vem sendo muito discutido, e que pouco ainda se sabe, pois são estudos recentes, é importante que se tenha uma análise bibliométrica sobre estudos dessa temática. Análise bibliométrica é considerada um estudo rápido e eficiente para avaliar as tendências de pesquisa e as redes acadêmicas em diferentes disciplinas de pesquisa (Zhang et al., 2019b; Zhang et al., 2016), podendo também fornecer orientação para pesquisadores jovens e iniciantes (Zhang et al., 2019b). Também pode encorajar e desafiar os pesquisadores a conduzir estudos adicionais (Tan et al., 2014). Na maioria dos casos, uma análise bibliométrica resume as tendências de pesquisa e redes acadêmicas com base em resultados de publicações, categorias de assunto, principais periódicos, autores ativos, países produtivos, instituições de pesquisa e frequências de palavraschave. Portanto, o objetivo deste estudo foi realizar uma análise bibliométrica e apresentar uma visão geral das tendências de 
pesquisa sobre a variabilidade espacial e temporal da vegetação de altitude em resposta às mudanças climáticas com o auxílio de geotecnologias, e elucidar os principais pontos de direções de pesquisas futuras.

\section{Metodologia}

\subsection{Estratégia de pesquisa e extração de dados}

Neste estudo, a pesquisa bibliográfica foi realizada usando o banco de dados Web of Science (WOS, https://clarivate.com), em 11 de dezembro de 2020, para reunir publicações acadêmicas sobre alterações espaço-temporal de vegetação de altitude em resposta às mudanças climáticas para os anos disponíveis. Este banco de dados é uma das fontes mais populares entre pesquisadores e acadêmicos e tem sido projetado especificamente para avaliar a importância e a influência das publicações científicas por meio do fator de impacto do periódico, apesar desse sistema ser um assunto de debate contínuo (Chiu \& Ho, 2005; Zhang et al., 2016). De acordo com o Journal Citation Reports (JCR) de 2018, o ISI Web of Science indexa 11.655 periódicos principais com referências de citação em 234 disciplinas científicas (Zhang et al., 2019).

Pesquisaram-se todas as publicações relacionadas ao tema desse estudo no banco de dados do ISI (Institute for Scientific Information) Web of Science Core Collection (Índices: SCI-EXPANDED, SSCI, A\&HCI, CPCI-S, CPCI-SSH, ESCI) utilizando os termos ("HIGH-ALTITUDE" OR "GRASSLAND" OR "HIGHLANDS" OR "VEGETATION" OR "ALPINE" OR "PASTURES") AND ("CLIMATE CHANGE" OR "CLIMATE VARIABILITY" OR "GLOBAL WARMING") AND ("REMOTE SENSING" OR "GEOPROCESSING" OR "REMOTE DETECTION") pelo campo do tópico que inclui título, resumo, palavras-chave do autor, e palavra-chave mais termos. No campo "tipos de documento", a busca foi filtrada para artigos, livros e capítulos de livros, escritos e publicados em inglês até 2020. Essa filtragem resultou em 3.081 documentos (Quadro 1).

Quadro 1 - Informações usadas como critério no refinamento da busca por artigos.

\begin{tabular}{|c|c|}
\hline Critério & Definição \\
\hline \multicolumn{2}{|l|}{ Bases de dados } \\
\hline & Web of Science (WOS) \\
\hline Índices & SCI-EXPANDED, SSCI, A\&HCI, CPCI-S, CPCI-SSH, ESCI \\
\hline Tipo de documentos & Artigos, Livros e Capítulos de Livros \\
\hline Strings (palavras-chave) & $\begin{array}{c}\text { ("HIGH-ALTITUDE" OR "GRASSLAND" OR "HIGHLANDS" OR } \\
\text { "VEGETATION" OR "ALPINE" OR "PASTURES") AND ("CLIMATE } \\
\text { CHANGE" OR "CLIMATE VARIABILITY" OR "GLOBAL WARMING") AND } \\
\text { ("REMOTE SENSING" OR "GEOPROCESSING" OR "REMOTE } \\
\text { DETECTION") }\end{array}$ \\
\hline Temática & $\begin{array}{c}\text { Variação espaço-temporal da vegetação de altitude em função das alterações } \\
\text { climáticas }\end{array}$ \\
\hline Período de publicação & 1945 a 2020 \\
\hline Instituição de pesquisa & Sem restrições \\
\hline Idioma de publicação & Inglês \\
\hline $\begin{array}{l}\text { Quantidade de documentos } \\
\text { filtrados }\end{array}$ & 3.081 \\
\hline
\end{tabular}

Fonte: Autores.

\subsection{Análise e visualização de dados}

Após a obtenção dos dados brutos da pesquisa bibliográfica (3.081 documentos), foi inicialmente realizada uma revisão dos documentos encontrados no software EndNote X9 (The EndNote Team, 2013), a partir da leitura dos títulos, palavras-chave 
e do resumo. Os artigos que tinham alguma relação com o campo de estudo em foco foram selecionados e, para garantir a precisão da busca e minimizar quaisquer erros dos dados fornecidos pelo banco de dados, foram examinados todos os documentos recuperados por meio de leitura integral, a fim de identificar publicações que não foram relacionadas ao campo de estudo, ou por falta de informação nos dados que se deseja analisar.

\subsection{Análise quantitativa}

A análise bibliométrica foi realizada usando uma abordagem de análise quantitativa e técnica de mapeamento de conhecimento. A análise quantitativa foi realizada com base nas informações fornecidas pela Web of Science, do crescimento e da citação de publicações, autores ativos, anos de publicação, palavras-chave do autor, países, revistas mais publicadas e áreas de conhecimento.

O mapeamento do conhecimento, ou seja, análise de rede, foi realizado usando-se o software VOSviewer (Van Eck; Waltman, 2010), onde visou a rede e a "força da ligação" entre palavras-chave do autor, países e autores.

\subsection{Análise qualitativa}

Para a análise qualitativa foi realizada a leitura integral dos documentos de maior relevância segundo o critério do número de citações e o "índice H" (H-index). Esse índice corresponde à quantidade de artigos com citações superior ou igual ao número de publicações de determinado autor, dando uma ideia de quais autores, numericamente, possuem maior nível de produção científica (Hirsch, 2005). Esse índice pode ser usado na estimativa do nível de impacto, de significância e de produtividade de autores e países (Chaves, 2020). Dentre os documentos que foram analisados, foi verificado o $\mathrm{H}$-index entre os autores e, assim, registrado o valor daquele de maior pontuação e deixando o nome sublinhado para identificação. Para melhor visualização do processo foi confeccionado um fluxograma descritivo representado na Figura 1. 
Figura 1 - Passo a passo ilustrado no fluxograma, onde os processos realizados na busca de artigos para a análise bibliométrica seguiram os critérios descritos nessa figura desde a base de dados (Web of Science) até o tratamento dos dados no EndNote. Fluxograma confeccionado gratuitamente no site Canva (Canva, 2020).

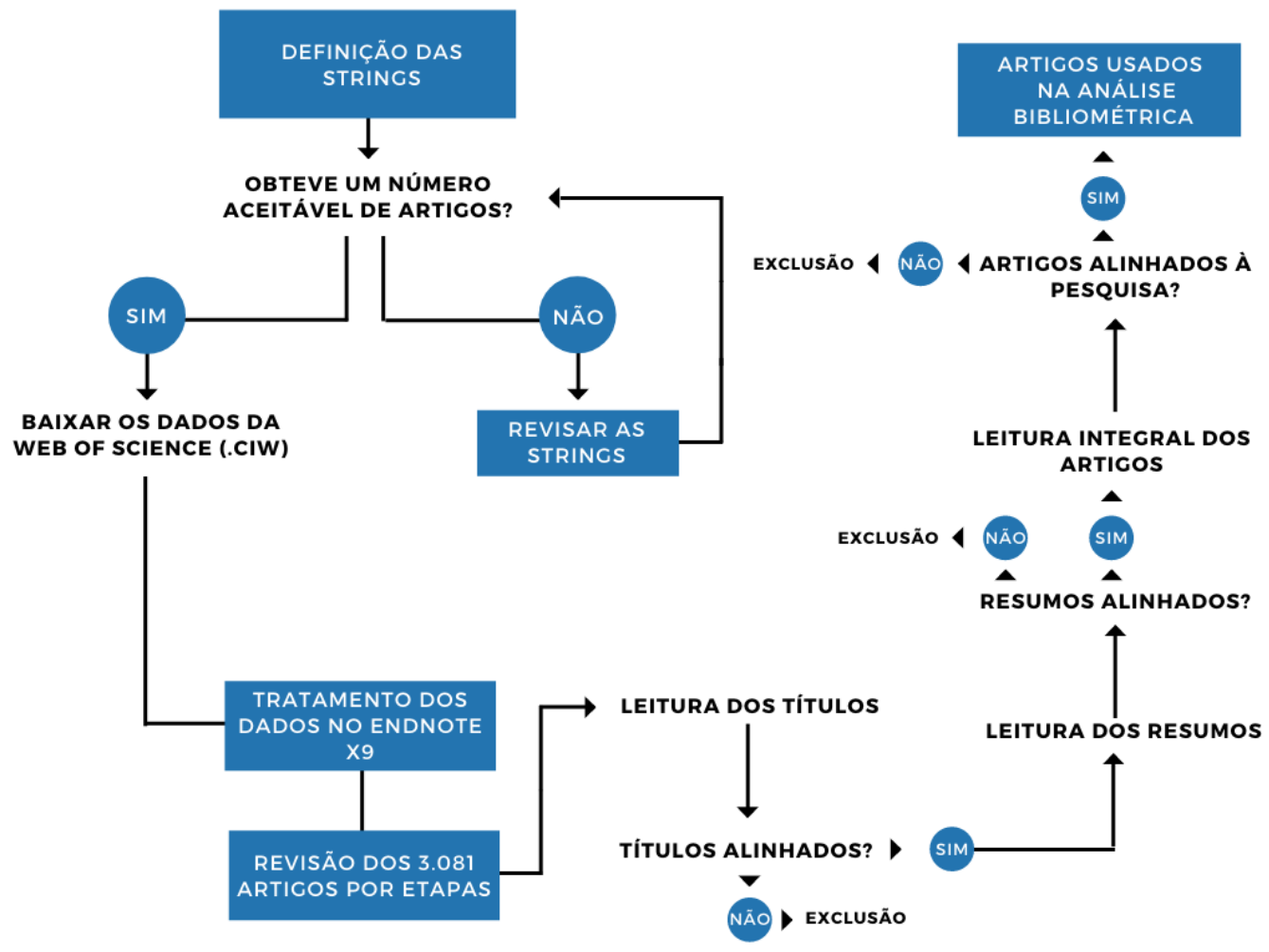

Fonte: Autores.

\section{Resultados e Discussão}

\subsection{Análise quantitativa: produção científica, e anos de publicação}

Obteve-se um total de 54 publicações com resumos alinhados. Foi realizada a leitura de todos esses documentos e chegou-se ao número de 27 publicações que atenderam a todos os critérios relacionados ao objetivo dessa análise (Figura 2).

Figura 2 - Quantidade de publicações por ano dos documentos alinhados à pesquisa.

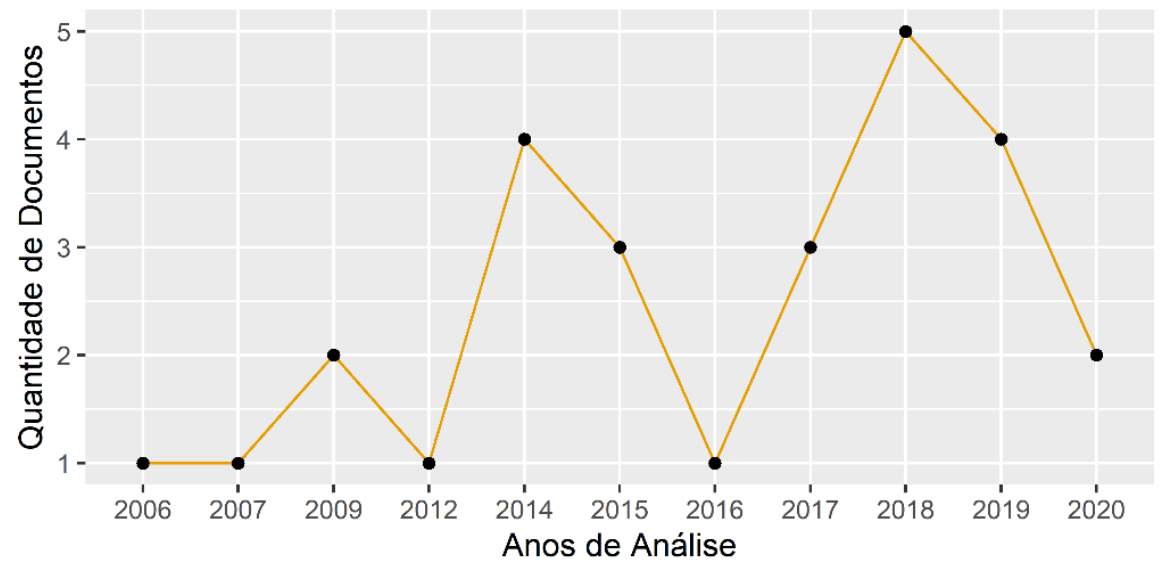

Fonte: Autores. 
Destes 27 documentos destacam-se os artigos com 21 (78,78\%), seguido de capítulos de livro com 5 (18,52\%) e livro com 1 (3,70\%). A primeira publicação nesse sentido foi registrada em 2006 e intitulada "Impact of climate change in the Hengduan Mountains of northwestern Yunnan, PR China: Vegetation distribution change in foretime and future" (Sun et al., 2006), na qual os autores realizaram uma simulação futura da distribuição espacial da vegetação de altitude nas montanhas de Hengduan, na China, usando dados climáticos. A última publicação foi intitulada "Climate warming benefits alpine vegetation growth in Three-River Headwater Region, China" (Bai et al., 2020)

O número de publicações dedicadas à análise espaço-temporal da vegetação de altitude, com o uso de sensoriamento remoto, aumentou a partir de 2014 e teve um déficit em 2016, tornando a ter maiores números de publicação a partir de 2017. Em 2020 o número foi baixo, o que pode estar relacionado aos artigos que ainda não haviam sido publicados até a data dessa análise. A mudança no número de documentos publicados é um índice importante na medição da pesquisa e do progresso de um determinado campo de assunto. Isso pode refletir o foco de atenção durante um determinado período, como um indicador de quais são os problemas que estão recebendo maior atenção por parte da academia científica (Wang et al., 2019).

\subsection{Os países e publicações mais citadas}

O número de publicações por países e o número de citações por ano é mostrado na Tabela 1.

Tabela 1 - Produção científica dos 5 principais países contribuintes.

\begin{tabular}{|c|c|c|c|c|c|c|c|c|c|c|}
\hline \multirow[b]{3}{*}{ Ano de Publicação } & \multicolumn{10}{|c|}{ Países } \\
\hline & \multicolumn{2}{|c|}{ China } & \multicolumn{2}{|c|}{ Romênia } & \multicolumn{2}{|c|}{ Alemanha } & \multicolumn{2}{|c|}{ Itália } & \multicolumn{2}{|c|}{ Eritreia } \\
\hline & NP & NC & NP & NC & NP & NC & $\mathbf{N P}$ & NC & NP & NC \\
\hline 2006 & 1 & 0 & & & & & & & & \\
\hline 2007 & 1 & 0 & & & & & & & & \\
\hline 2009 & 2 & 91 & & & & & & & & \\
\hline 2012 & 1 & 1 & & & & & & & & \\
\hline 2014 & 4 & 55 & & & & & & & & \\
\hline 2015 & 3 & 75 & & & & & & & & \\
\hline 2016 & 1 & 39 & & & & & & & & \\
\hline 2017 & 2 & 23 & 1 & 15 & & & & & & \\
\hline 2018 & 4 & 26 & & & 1 & 7 & & & & \\
\hline 2019 & 2 & 4 & & & & & 1 & 4 & 1 & 10 \\
\hline 2020 & 2 & 0 & & & & & & & & \\
\hline Total & 23 & 314 & & & & & & & & \\
\hline
\end{tabular}

NP: Número de Publicações; NC: Número de Citações. Fonte: Autores.

Pode-se observar que o número de publicações ao longo dos primeiros anos foi majoritariamente realizado por pesquisadores da China, ocorrendo uma publicação em 2017 por pesquisadores da Romênia e seguido de outros países nos anos subsequentes. O número de artigos publicados por pesquisadores chineses foi maior do que o publicado por outros pesquisadores de outras nações, sendo que só em 2017 ocorreu o interesse de pesquisas por parte de outras nações no monitoramento da dinâmica espaço-temporal da vegetação de altitude em resposta às mudanças climáticas com o uso de sensoriamento remoto. Vale ressaltar que há mais publicação por parte dos chineses que de outros pesquisadores de outras nações em todos os anos de 
análise. Os dados sugerem que os estudiosos chineses estão se tornando mais favoráveis a publicar dados que corroboram com as alterações climáticas do que outras nações, e para uma exposição mais ampla de suas pesquisas estão sendo publicados em língua inglesa em periódicos internacionais, aumentando suas colaborações internacionais.

O aumento de publicações na área de mudanças climáticas, por parte de pesquisadores da China, pode ter relação com acordos de cooperação internacional sobre mudanças climáticas, do qual a China iniciou mudanças significativas em muitos aspectos ( $\mathrm{Li}, 2016)$. Paralelamente às suas conquistas econômicas, o volume de publicações científicas oriundos da China, no monitoramento ambiental, também aumentou exponencialmente, e ultrapassou os Estados Unidos como o maior colaborador de artigos científicos na área de monitoramento ambiental (Engels, 2018; Xie, 2020). Sob a perspectiva dos líderes da China foram traçadas mudanças significativas por um sistema de gestão mais ecológica e ambiental mais eficiente, visando realizar um arranjo institucional na busca de um estágio de desenvolvimento de conceitos de governança que devem levar em conta o crescimento do país, mas que também se preocupe com a proteção ambiental, o que significa que as autoridades competentes em matéria de recursos, proteção ecológica e ambiental deve desempenhar um papel mais importante, aumentando dessa forma os investimento em matéria de desenvolvimento de pesquisas que monitorem as alterações climáticas e do meio ambiente (Xie, 2020).

\subsection{Periódicos mais ativos, áreas do conhecimento e palavras-chave}

A análise dos periódicos permite obter uma visão geral do tema em estudo nos diversos campos acadêmicos, assim, na Tabela 2 são apresentados todos os periódicos que registram o número de publicações sobre o assunto dessa pesquisa; além disso, o número de citações recebidas, o fator de impacto (CiteScore 2019) e o Scimago Journal Ranking (SJR) também estão disponíveis. Ao todo foram 22 periódicos, do total de 27 publicações, tendo um total de citações de 367 (Figura 3). O periódico mais citado é o Climatic Change, com 1 artigo; 94 citações (23,44\%), Citescore: 8.3; SJR: 1.908, seguido por National Science Review, com 1 artigos; 52 citações (12,97\%), Citescore: 9.1; SJR: 2.243, e Science of the Total Environment, com 3 artigos; 44 citações (10,97\%); Citescore: 8.6; SJR: 1.661. 
Tabela 2 - Ranking dos periódicos com maior produtividade científica e número de citações. NP: Número de Publicações; NC: Número de Citações; NC\%: Percentual do Número de Citações.

\begin{tabular}{cccccc}
\hline Periódico & NP & NC & NC\% & $\begin{array}{c}\text { Citescore } \\
\text { So19 }\end{array}$ & SJR \\
\hline Climatic Change & 1 & 94 & 23,44 & 8.3 & 1.908 \\
National Science Review & 1 & 52 & 12,97 & 9.1 & 2.243 \\
Science of the Total Environment & 3 & 44 & 10,97 & 8.6 & 1.661 \\
Sensors & 1 & 34 & 8,48 & 5.0 & 0.653 \\
Agricultural and Forest Meteorology & 1 & 26 & 6,48 & 7.2 & 1.836 \\
Quaternary International & 1 & 23 & 5,74 & 4.9 & 1.015 \\
Remote Sensing of Environment & 1 & 23 & 5,74 & 15.1 & 3.541 \\
International Journal of Remote Sensing & 1 & 21 & 5,24 & 4.6 & 0.928 \\
Environmental Research Letters & 1 & 20 & 4,99 & 8.9 & 2.675 \\
Climate Research & 1 & 17 & 4,24 & 4.0 & 0.804 \\
Remote Sensing & 1 & 13 & 3,24 & 6.1 & 1.422 \\
Biology Letters & 1 & 11 & 2,74 & 5.6 & 1.651 \\
Biogeosciences & 1 & 7 & 1,75 & 6.7 & 1.761 \\
Applied Vegetation Science & 1 & 4 & 1,00 & 6.0 & 1.115 \\
Ecological Indicators & 1 & 4 & 1,00 & 7.6 & 1.331 \\
Environmental Earth Sciences & 1 & 4 & 1,00 & 4.0 & 0.604 \\
Canadian Journal of Remote Sensing & 1 & 2 & 0,50 & 4.9 & 0.905 \\
Scientific World Journal & 1 & 1 & 0,25 & 2.9 & 0.384 \\
Symposium (IGARSS) & 1 & 1 & 0,25 & 0.9 & 0.279 \\
Arabian Journal of Geosciences & 1 & 0 & 0,00 & 2.0 & 0.404 \\
Forests & 1 & 0 & 0,00 & 2.7 & 0.652 \\
Spie Int Soc Optical Engineering & 0 & 0,00 & 1.0 & 0.215 \\
\hline International Geoscience and Remote Sensing & 4 & & & & \\
\hline
\end{tabular}

Fonte: Autores. 
Research, Society and Development, v. 10, n. 8, e58710817657, 2021

(CC BY 4.0) | ISSN 2525-3409 | DOI: http://dx.doi.org/10.33448/rsd-v10i8.17657

Figura 3 - Número de citações por periódicos.

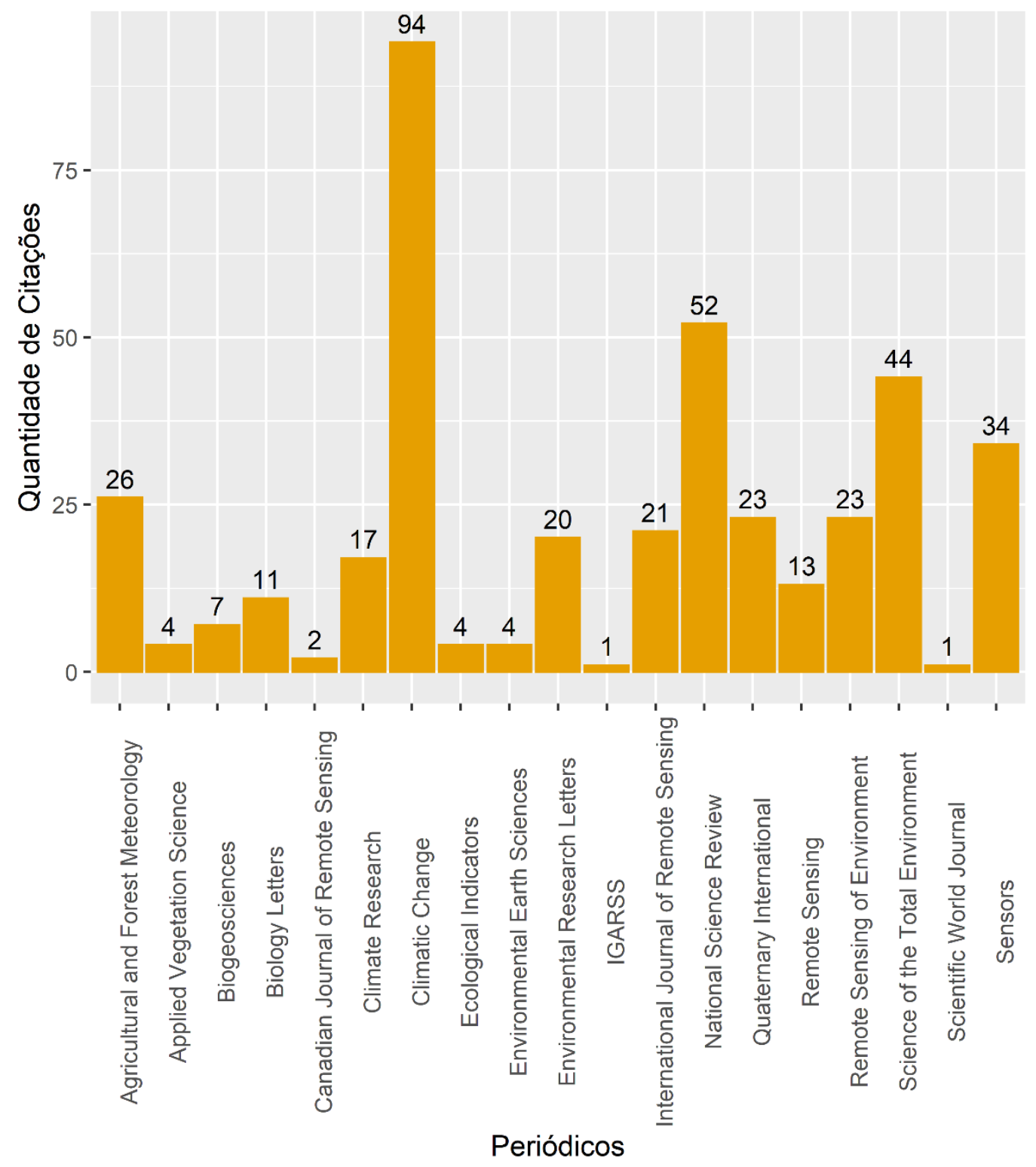

Fonte: Autores.

Verifica-se na Figura 4 que as citações das publicações aqui analisadas começam no ano de 2010 e possuem uma tendência de aumentar ao longo dos anos, principalmente a partir de 2016. 
Figura 4 - Número de vezes que os documentos analisados nessa pesquisa foram citados ao longo dos anos de análise.

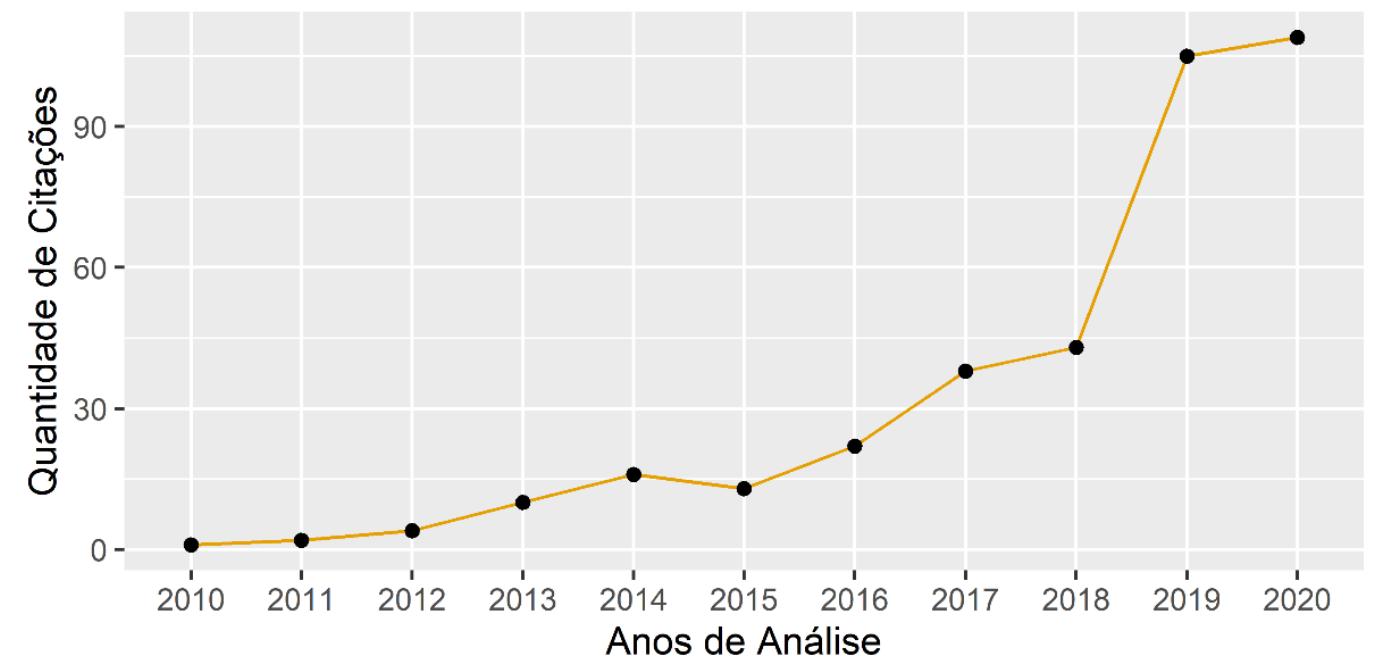

Fonte: Autores.

Isso sugere que tem aumentado o interesse de pesquisadores em desenvolvimento de pesquisas na área de monitoramento das alterações climáticas e seus efeitos sobre a vegetação, pois como é verificado nos artigos aqui analisados, tiveram eles objetivos similares aos da pesquisa aqui desenvolvida, ou seja, possuem alguma relação na dinâmica de clima $\mathrm{x}$ vegetação.

O crescimento exponencial da literatura sobre mudanças climáticas, de maneira geral, e de áreas específicas, pode ser resultado de vários fatores, como por exemplo, os encontros entre as nações para discutir as mudanças climáticas, também possivelmente induzido pela crescente influência dos Relatórios de Avaliação do IPCC que, eventualmente, tornaram a pesquisa sobre mudanças climáticas um dos temas mais importantes atualmente (Haunschild et al., 2016). Além disso, os efeitos, impactos e riscos das mudanças climáticas sobre a biosfera tornaram-se uma preocupação cada vez mais concreta, o que induz à maioria da comunidade científica a realizar pesquisas sobre esse tema (Tai \& James, 2018; Haunschild et al., 2016).

A pesquisa por área de conhecimento cobriu 19 áreas temáticas (Figura 5) identificadas pelo banco de dados usado nessa pesquisa (WOS, Web of Science). As quatro categorias mais comuns foram Ciências Ambientais com 9 publicações indexadas nessa área (17,65\%), Sensoriamento Remoto com 8 publicações $(15,69 \%)$, Geociências com 6 publicações (11,76\%) e Ciências Meteorológicas Atmosféricas com quatro (4) publicações (7,84\%). A Figura 6 apresenta a produção científica desses quatro grupos em porcentagem, por área temática. Percebe-se, assim, o amplo campo de pesquisa, em diversas áreas, que propõe o tema em análise. 
Research, Society and Development, v. 10, n. 8, e58710817657, 2021

(CC BY 4.0) | ISSN 2525-3409 | DOI: http://dx.doi.org/10.33448/rsd-v10i8.17657

Figura 5 - Produção científica por área de conhecimento.

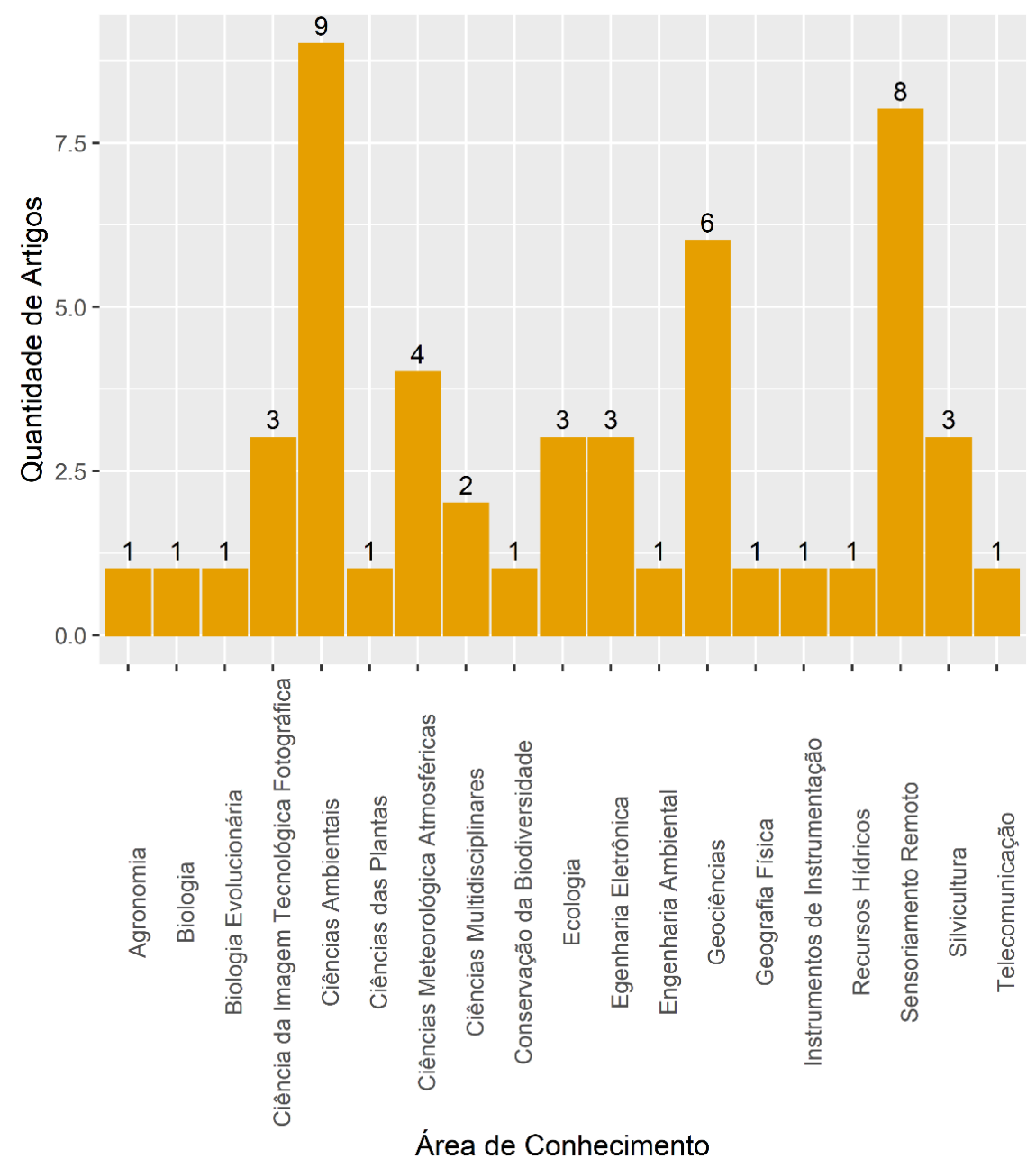

Fonte: Autores.

Figura 6 - Percentual por área de conhecimento.

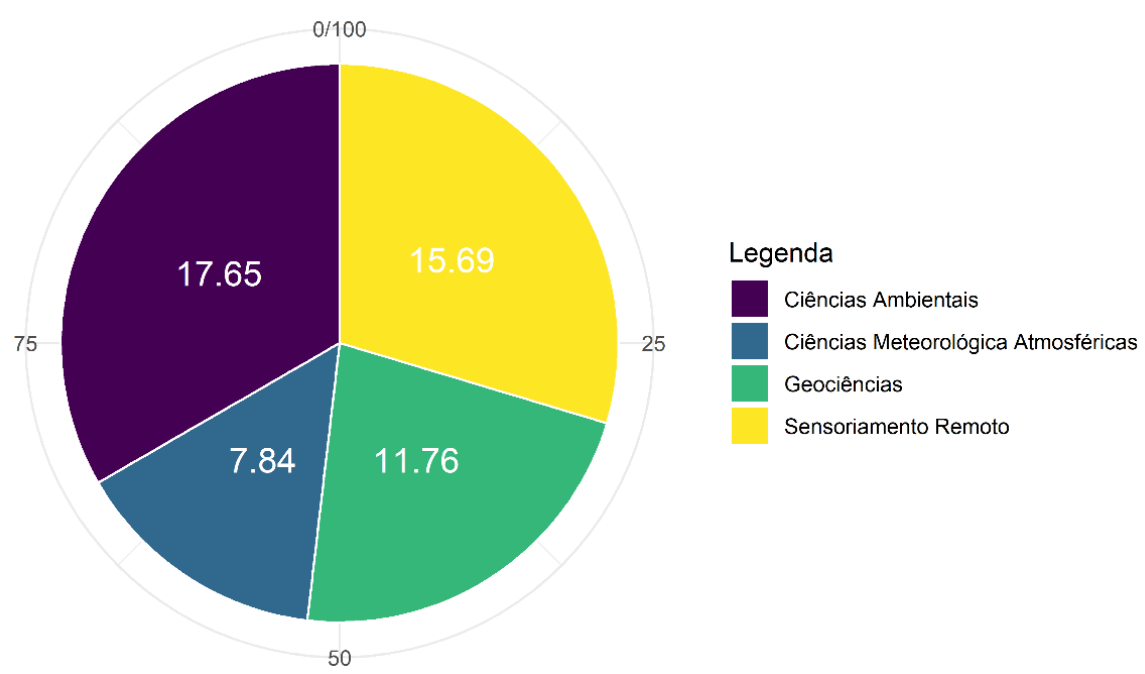

Fonte: Autores. 
As palavras-chave podem fornecer indicações sobre as prioridades de pesquisa e os interesses dos cientistas e pesquisadores em determinadas áreas de pesquisa. De acordo com essas palavras-chave, os dez primeiros, em termos de ocorrências totais, estão descritos na Tabela 3. As palavras-chave Climate Change, Remote Sensing e Tibetan Plateau tiveram maior frequência de uso nas publicações analisadas.

Tabela 3 - As 10 principais palavras-chave de alta frequência das publicações analisadas nessa pesquisa.

\begin{tabular}{ccc}
\hline Número & Palavras-chave & $\begin{array}{c}\text { Frequênci } \\
\text { a }\end{array}$ \\
\hline 1 & Climate Change & 15 \\
2 & Remote Sensing & 14 \\
3 & Tibetan Plateau & 12 \\
4 & NDVI & 8 \\
5 & Temperature & 7 \\
6 & Vegetation & 6 \\
7 & China & 6 \\
8 & Ecosystems & 5 \\
9 & MODIS & 5 \\
10 & Time-series & 4 \\
\hline
\end{tabular}

Fonte: Autores.

A análise da relação entre as palavras-chave e as suas forças de ligação é usada para determinar a estrutura cognitiva e os tópicos mais importantes do campo acadêmico e das áreas estudadas, usando mapas visuais de semântica, com base na análise de conteúdo técnico (Dong \& Chen, 2015; Herrera-Franco et al., 2020). O software VOSviewer (Van Eck; Waltman, 2010) foi utilizado para a construção da co-ocorrência das palavras-chave, usando as palavras-chave do autor do banco de dados obtido da Web of Science. As palavras-chave NDVI, Remote Sensing, Climate Change, Temperature e Tibetan Plateau tiveram as maiores forças de ligação entre todas as palavras-chave do autor e estavam altamente conectadas a Remote Sensing (Figura 7). Além disso, essas informações relacionadas à Força Total do link (força de ligação) denota a importância de uma palavra-chave no campo, uma vez que um valor mais alto significa que ela foi vinculada a outras palavras-chave mais vezes. As palavras-chave Dynamics, Trend Analysis e MODIS foram as mais utilizadas atualmente, conforme a escala em anos, na cor amarela, mais próxima de 2020.

Vale ressaltar que, quando um grande número de palavras-chave do autor aparece uma única vez, mostra a falta de continuidade e divergência na pesquisa, enquanto que as palavras-chave possuem maior número de repetições permitem identificar os tópicos mais utilizados no campo de pesquisa e determinação de sua estrutura (Herrera-Franco et al., 2020; BaierFuentes et al., 2019). Essas palavras-chave são usadas pelos autores para descrever o mesmo conceito, por isso é necessário estabelecer um padrão de consistência em sua estrutura, exigindo o ajuste dos termos encontrados no plural para o singular e sinônimos antes da construção das strings que serão usados na busca por artigos nas bases de dados. 
Research, Society and Development, v. 10, n. 8, e58710817657, 2021

(CC BY 4.0) | ISSN 2525-3409 | DOI: http://dx.doi.org/10.33448/rsd-v10i8.17657

Figura 7 - Análise de rede de co-ocorrência de palavras-chave do autor mais frequentemente usadas. As cores são representadas na legenda de anos (2014-2018).

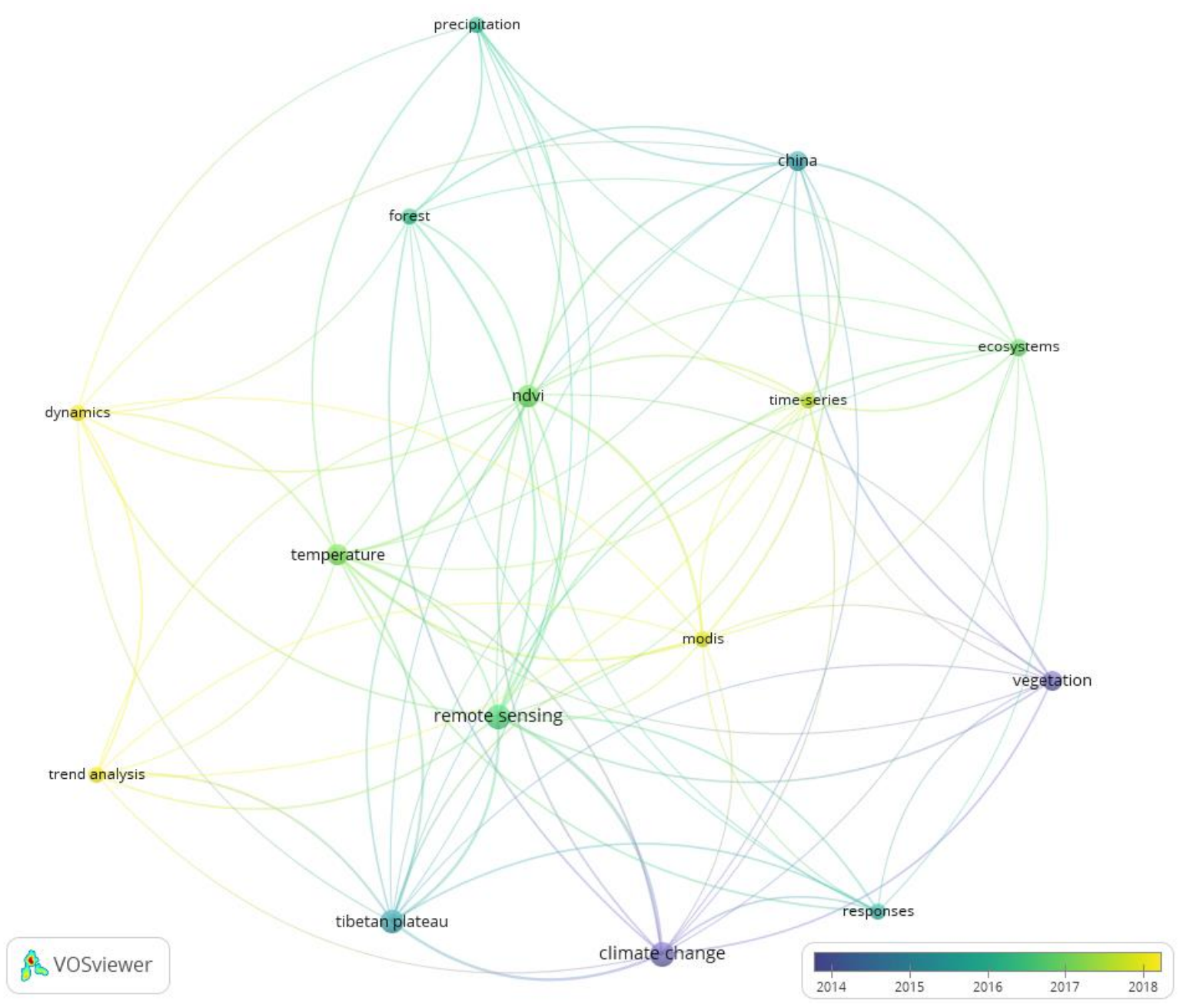

Fonte: Autores.

\subsection{Análise qualitativa: autores mais ativos}

Para cada um dos dez artigos foi registrado o H-index do autor com a pontuação mais alta e sublinhado o nome para identificação (Tabela 4). 
Tabela 4 - Informações bibliográficas dos dez autores com maior H-index entre os artigos usados nessa análise.

\begin{tabular}{|c|c|c|}
\hline Número & Artigo & H-index \\
\hline 1 & $\begin{array}{l}\text { He, Z. B., et al. (2015). Assessing temperature sensitivity of subalpine shrub phenology in semi- } \\
\text { arid mountain regions of China. Agricultural and Forest Meteorology 213: } 42-52\end{array}$ & 32 \\
\hline 2 & $\begin{array}{l}\text { Zhou, L. H., et al. (2009). Response of Vegetation Coverage on Climate Change in Arid } \\
\text { Mountain of Northwest China. } 2009 \text { Ieee International Geoscience and Remote Sensing } \\
\text { Symposium, Vols 1-5. New York, Ieee: 1871- }\end{array}$ & 28 \\
\hline 3 & $\begin{array}{l}\text { Li, W. L., et al. (2012). Typical alpine wetland landscape changes in eastern tibetan plateau } \\
\text { under climate change over } 15 \text { years. } 2012 \text { Ieee International Geoscience and Remote Sensing } \\
\text { Symposium. New York, Ieee: } 4907-4910 .\end{array}$ & 26 \\
\hline 4 & $\begin{array}{l}\text { Shen, M. G., et al. (2015). Plant phenological responses to climate change on the Tibetan } \\
\text { Plateau: research status and challenges. National Science Review 2(4): 454-467. }\end{array}$ & 25 \\
\hline 5 & $\begin{array}{l}\text { Huang, N., et al. (2018). No upward shift of alpine grassland distribution on the Qinghai-Tibetan } \\
\text { Plateau despite rapid climate warming from } 2000 \text { to } 2014 \text {. Science of the Total Environment } \\
\text { 625: 1361-1368. }\end{array}$ & 21 \\
\hline 6 & $\begin{array}{l}\text { Zhou, H. K., et al. (2014). Field evidence for earlier leaf-out dates in alpine grassland on the } \\
14 \text { mpacto Tibetan Plateau from } 1990 \text { to } 2006 \text {. Biology Letters 10(8): } 5 \text {. }\end{array}$ & 19 \\
\hline 7 & $\begin{array}{l}\text { Sun, J. H., et al. (2006). 14mpacto f climate change in the Hengduan Mountains of northwestern } \\
\text { Yunnan, PR China: Vegetation distribution change in foretime and future. Earth Observing } \\
\text { Systems Xi. J. J. Butler and J. Xiong. Bellingham, Spie-Int Soc Optical Engineering. } 6296 .\end{array}$ & 17 \\
\hline 8 & $\begin{array}{l}\text { Gao, Q. Z., et al. (2009). Dynamics of alpine grassland NPP and its response to climate change } \\
\text { in Northern Tibet. Climatic Change 97(3-4): 515-528. }\end{array}$ & 16 \\
\hline 9 & $\begin{array}{l}\text { Li, C. B., et al. (2014). Regional vegetation dynamics and its response to climate change-a case } \\
\text { study in the Tao River Basin in Northwestern China. Environmental Research Letters 9(12): } 12 \text {. }\end{array}$ & 14 \\
\hline 10 & $\begin{array}{l}\text { Zhang, J., et al. (2007). Evaluation of Grassland Dynamics in the Northern-Tibet Plateau of } \\
\text { China Using Remote Sensing and Climate Data. Sensors, 7:3312-3328. }\end{array}$ & 13 \\
\hline
\end{tabular}

Fonte: Autores.

É importante compreender melhor a extensão e a gravidade dos impactos das mudanças climáticas sobre a vegetação de altitude e, ao usar uma abordagem bibliométrica para revisar a literatura sobre os efeitos do clima sobre as respostas da vegetação de altitude, no espaço e tempo, com o uso de ferramentas de sensoriamento remoto, foi possível identificar os principais autores que tratam desse assunto e as publicações que sustentam essa literatura, visando entender melhor quais trabalhos estão sendo realizados nesse sentido. Os próximos parágrafos discutem a diversidade, a complexidade, as aplicações e a amplitude da pesquisa, as suas implicações e lacunas, bem como os benefícios e as limitações desta metodologia de revisão.

O trabalho desenvolvido por He et al. (2015) implicou em entender as alterações fenológicas da vegetação de altitude, ou seja, a distribuição dos tipos de vegetação arbustivos alpinos em resposta à mudanças de temperatura numa região montanhosa da China e, a partir dessas observações, entender as restrições climáticas no tempo de folhagem e foliação, além das alterações espaciais desse tipo de vegetação e projetar padrões fenológicos em diferentes cenários futuros, quando possa ocorrer mudanças climáticas mais acentuadas. O trabalho vale-se de uma ferramenta comumente utilizada para realizar este tipo de observação, que é o índice de vegetação, geralmente usa-se o NDVI (Normalized Difference Vegetation Index), que possui mais aplicabilidade sobre a vegetação e é um índice atraente devido a sua capacidade de delinear rapidamente a vegetação e o estresse vegetativo, o que tem grande apelo em trabalhos ambientais e em estudos de uso da terra. Essa técnica foi rapidamente reconhecida na comunidade científica no início dos anos 1970 e todos os dados de sensoriamento remoto de satélite de observação da Terra foram equipados para produzir este índice em diferentes resoluções espaciais e temporais (Huang, et al., 2020).

O Índice de Vegetação por Diferença Normalizada (NDVI), usado na pesquisa de He et al. (2015), foi utilizado para relacionar as alterações da escala de NDVI e entender a dinâmica da vegetação de altitude em relação à temperatura. Já as 
pesquisas desenvolvidas por Li et al. (2014), Shen et al (2015) e Zhang et al. (2007) buscaram entender também as dinâmicas da vegetação de altitude da região do Planalto Tibetano, na China e relacionaram alguns índices de vegetação para visualização da distribuição espaço-temporal desses índices, entre eles o índice de vegetação NDVI, com as variáveis de temperatura e precipitação ao longo de uma série temporal para determinar se o clima é ou não o motor dominante da mudança na vegetação de altitude e, em caso afirmativo, quais são as variáveis climáticas específicas que são mais eficazes para prever a mudança na distribuição da vegetação de altitude. Sun et al. (2006) e Zhou et al. (2009) aplicaram o índice de vegetação NDVI ao longo de uma região montanhosa da China com presença de vegetação de altitude e relacionaram as variações espaciais do NDVI ao longo de uma série histórica com variáveis climáticas para entender se havia alguma conexão entre as diferenças espaciais e temporais do índice com as flutuações das variáveis climáticas.

Também foram realizadas outras relações do uso do NDVI na busca de resultados que relacionassem as variações da vegetação de altitude às alterações climáticas. A pesquisa desenvolvida por Huang et al. (2018) visou monitorar se a distribuição de vegetação de altitude aumentou em altitude no planalto tibetano e para tanto utilizou-se de uma análise de NDVI e cálculos de biomassa vegetal, dimensionando a distribuição vegetal no perfil altimétrico do planalto tibetano, na China, ao longo de uma série temporal, para tentar entender se há alguma relação com fatores externos, como por exemplo, as variações climáticas. Há pesquisas na literatura que se utilizam de técnicas de medição de biomassa vegetal através de índices de vegetação, como é o caso do NDVI, pois há fortes relações exponenciais entre NDVI e biomassa, NDVI e LAI (Leaf Area Index), que sugerem que o NDVI satura para valores mais altos de biomassa (Huete et al., 2002; Santin-Janin et al., 2009; Walker et al., 2005; Boelman et al., 2003). Portanto, trabalhos que se utilizam de cálculos de NDVI na estimativa de biomassa vegetal são importantes no sentido de visualizar se uma determinada vegetação sofreu redução espacial numa determinada área, e a biomassa é um forte indicador, uma vez que alterações de biomassa sugerem que houve substituição de uma vegetação menor por outro maior ou vice-versa (Huang et al., 2018).

Outro trabalho interessante foi desenvolvido por Gao et al. (2009), que utilizou índices de vegetação e analisou a produtividade primária líquida da vegetação de altitude no planalto tibetano e a sua dinâmica em resposta às mudanças climáticas. Essa análise da produtividade foi realizada dentro de uma série histórica (1981 a 2004) e, para tanto, utilizou-se do NDVI para aplicar uma fórmula da estimativa da produção primária líquida (NPP, Net Primary Productivity) para depois correlacionar a NPP com elementos climáticos de temperatura, precipitação, radiação solar e visualizar se há uma relação da variação de NPP com as flutuações dos dados climáticos. Esse trabalho de análise da produção primária líquida da vegetação de altitude é extremamente importante em estudos de mudanças ambientais em níveis globais, no que diz respeito à identificação dos impactos das mudanças climáticas na produção primária líquida da vegetação de altitude, e há trabalhos de pesquisas anteriores que estudaram a relação entre as mudanças climáticas e a dinâmica da produção primária líquida da vegetação em escalas global e regional (Gao et al., 2009; Melillo et al., 1993; Knapp \& Smith, 2001; Fang et al., 2001; Nemani et al., 2003), e com isso entender melhor o comportamento da variação espacial da vegetação em resposta à variações exógenas (Gao et al., 2009).

\section{Considerações Finais}

Neste estudo apresentaram-se as tendências globais de pesquisas e redes acadêmicas sobre monitoramento das alterações espaço-temporal da vegetação de altitude em resposta às mudanças climáticas, por meio do sensoriamento remoto, cobrindo o período de 1945 a 2020.

No geral, os resultados bibliométricos deste estudo forneceram uma avaliação quantitativa do desenvolvimento da pesquisa no campo do monitoramento das mudanças espaço-temporal de vegetação de altitude usando sensoriamento remoto. Os resultados são um resumo das publicações em grande parte por acadêmicos chineses. Além de enriquecer o entendimento sobre o desenvolvimento histórico, status atual e tendência destas pesquisas, na China e no mundo todo, este estudo verificou o 
esforço colaborativo entre acadêmicos chineses de uma ampla gama de organizações no monitoramento de mudanças climáticas e seus efeitos sobre a flora de altitude. Além disso, os resultados deste estudo podem auxiliar estudantes e pesquisadores nas suas pesquisas e projetos, assim como a se envolver em esforços conjuntos de pesquisa em campos relacionados.

A análise demonstrou ainda que o número de publicações relacionadas ao tema desta pesquisa ainda é muito pequeno e restrito a poucos países e a tipos de vegetação específicas de determinadas áreas alpinas. A maioria das pesquisas foram desenvolvidas por pesquisadores chineses, sendo que a área mais estudada, nesse campo de pesquisa, foi o planalto tibetano na China. Isso indica que há escassez de estudos em outras regiões e países com diferentes tipos de vegetação e fatores climáticos regionais diferentes, o que torna necessário haver pesquisas em outros locais com condições diferentes, no que tange ao tipo de vegetação de altitude e aos fatores climáticos, na busca por fornecer informações precisas e oportunas sobre as alterações espaçotemporal da vegetação de altitude que é um tipo de vegetação sensível às mudanças climáticas, sendo uma ótima forma de buscar levantar informações quanto aos efeitos das alterações do clima na flora regional.

Além do mais, este estudo verificou que não há estudos publicados desenvolvidos no Brasil, no que se refere ao monitoramento das alterações espaço-temporal do Campo de Altitude (vegetação de altitude regional) presente no Pantanal, este que é um importante hotspot mundial e por conta de que as áreas úmidas estão entre os ecossistemas mais frágeis e ameaçados da Terra, pois estão sujeitas ao impacto das atividades humanas e, mudanças climáticas poderiam ter graves impactos na hidrologia do Pantanal (Ioris et al., 2014; Junk et al., 2006), acarretando em graves consequências socioecológicas (Bergier, 2013). Também, mudanças nas condições climáticas na região podem causar perturbações significativas no funcionamento do ecossistema, principalmente por alterar as taxas de precipitação e evapotranspiração que, por sua vez, podem afetar o regime de fluxo do rio e a dinâmica de inundação da planície de inundação. Os impactos das mudanças climáticas podem até mesmo ampliar e agravar consequências indesejáveis de algumas intervenções humanas nas condições hidrológicas da bacia (Bravo et al., 2014; Junk et al., 2006).

Portanto, torna-se importante estudos que visem monitorar as condições climáticas no Pantanal, pois o Pantanal tem importância como sistema hidrológico de amortecimento para áreas da jusante, regulador climático regional, valiosa área de retenção e purificação de água e centro de manutenção da biodiversidade (Junk et al., 2006). Apesar de enfrentar as implicações atuais na legislação ambiental brasileira, é preciso dar atenção a este bioma sensível, e que as políticas dos tomadores de decisão levam em consideração a importância do Pantanal para o mundo. Visando a importância do desenvolvimento de estudos no monitoramento da dinâmica da vegetação Campo de Altitude no Pantanal, estudos são necessários visando compreender as dinâmicas espaço-temporal do Campo de Altitude presente no Pantanal que possam fornecer respostas do atual cenário ambiental desse importante hotspot.

\section{Agradecimentos}

Os autores agradecem ao Programa de Pós-Graduação em Tecnologias Ambientais (PPGTA), da Faculdades de Engenharias, Arquitetura e Urbanismo (FAENG) da Fundação Universidade Federal de Mato Grosso do Sul (UFMS).

O presente trabalho foi realizado com apoio da Fundação Universidade Federal de Mato Grosso do Sul - UFMS/MEC - Brasil.

O presente trabalho foi realizado com apoio da Coordenação de Aperfeiçoamento de Pessoal de Nível Superior - Brasil (CAPES) - Código de Financiamento 001.

Ao CNPq (Conselho Nacional de Desenvolvimento Científico e Tecnológico) pela bolsa de produtividade em pesquisa de A. C. Paranhos Filho (CNPq Processo 305013/2018-1).

Agradecemos a CAPES pelo acesso ao Portal de Periódicos.

Agradecemos ainda a CAPES pela bolsa de doutorado de Dhonatan Diego Pessi, processo número 88887.494036/2020- 
00.

\section{Referências}

Bluden, J. \& Arndt, D. S. (2018). State of the Climate in 2017. Bull. Am. Meteorol. Soc., 99, 332.

Bai, Y. et al. (2020). Climate warming benefits alpine vegetation growth in Three-River Headwater Region, China. Science of the Total Environment, 742, 140574.

Baier-Fuentes, H. et al. (2019). International entrepreneurship: A bibliometric overview. Int. EntreManag. J., 15, 385-429.

Boelman, N. T. et al. (2003). Response of NDVI biomass and ecosystem gas exchange to long-term warming and fertilization in wet sedge tundra. Oecologia, $135,414-421$.

Bergier, I. (2013). Effects of highland land-use over lowlandsof the Brazilian Pantanal. Sci Total Environ, 463-464, 1060-1066.

Bravo, J. M. et al. (2014). Impact of projected climate change on hydrologicregime of the Upper Paraguay River basin. Clim Change, 127, $27-41$.

CANVA. (2020). https://www.canva.com/pt_br/.

Chaves, J. R. (2020). Sintaxe Espacial e Mobilidade na Paisagem Urbana como ferramenta para gestão. Dissertação de Mestrado. Fundação Universidade de Mato Grosso do Sul, 125p.

Chiu, W. T \& Ho, Y. S. (2005). Bibliometric analysis of homeopathy research during the period of 1991 to 2003 . Scientometrics, 63, 3-23.

Correa-Díaz, A. et al. (2020). Long-Term Wood Micro-Density Variation in Alpine Forests at Central México and Their Spatial Links with Remotely Sensed Information. Forests, 11, 452.

Dong, D. \& Chen, M. L. (2015). Publication trends and co-citation mapping of translation studies between 2000 and 2015. Scientometrics, v. 105, 1111-1128.

Engels, A. (2018). Understanding how China is championing climate change mitigation. PALGRAVE COMMUNICATIONS, 4 (101), 1-6.

Gao, Q. et al. (2009). Dynamics of alpine grassland NPP and its response to climate change in Northern Tibet. Climate Change, 97, 515-528.

Haunschild, R.; Bornmann, L.; \& Marx, W. (2016). Climate Change Research in View of Bibliometrics. PLoS ONE, 11 (7), e0160393.

Huete, A. et al. (2002). Overview of the radiometric and biophysical performance of the MODIS vegetation indices. Remote Sensing of Environment, 83, 195213.

Huang, N. et al. (2018). No upward shift of alpine grassland distribution on the Qinghai-Tibetan Plateau despite rapid climate warming from 2000 to 2014. Science of the Total Environment, 625, 1361-1368.

Huang, S. (2020). A commentary review on the use of normalized difference vegetation index (NDVI) in the era of popular remote sensing. J. For. Res., 32, 16.

Herrera-Franco, G.; et al. (2020). Research Trends in Geotourism: A Bibliometric Analysis Using the Scopus Database. Geosciences, 10 (379), 1-29.

He, Z. B. et al. (2015). Assessing temperature sensitivity of subalpine shrub phenology in semi-arid mountain regions of China. Agricultural and Forest Meteorology, 213, 42-52.

Hirsch, J. E. (2005). An index to quantify an individual's scientific research output. Proceedings of the National academy of Sciences, 102 (46), 16569-16572.

Ioris, A. A. R.; Irigaray, C. T.; \& Girard, (2014). Institutionalresponses to climate change: opportunities and barriersfor adaptation in the Pantanal and the Upper ParaguayRiver Basin. Clim Change, 127, 139-151.

Fang, J. et al. (2001). Inter-annual variability in net primary production and precipitation. Science, 293, 1723a.

Junk, W. J. et al. (2006). Biodiversity and its conservation in the Pantanal of Mato Grosso, Brazil. Aquat. Sci. 68, $278-309$.

Knapp, A. K. \& Smith, M. D. (2001). Variation among biomes in temporal dynamics of aboveground primary production. Science, 291 , $481-484$.

Li, A. H. F. (2016). Hopes of Limiting Global Warming? China and the Paris Agreement on Climate Change. China Perspectives, 1 , $49-54$.

Li, C. B. et al. (2014). Regional vegetation dynamics and its response to climate change-a case study in the Tao River Basin in Northwestern China. Environmental Research Letters, 9 (12), 12.

Pepin, N. et al. (2015). Elevation-dependent warming in mountain regions of the world. Nat. Clim. Chang, 5, 424-430.

Ma, X. et al. (2019). Sensitivity of Vegetation on Alpine and Subalpine Timberline in Qinling Mountains to Temperature Change. Forests, 10 , 1105.

Melillo, J. M. et al. (1993). Global climate change and terrestrial net primary production. Nature, 363, 234-240.

Nemani, R. R. (2003). Climate-driven increases in global terrestrial net primary production from 1982 to 1999. Science, $300,1560-1563$. 
Research, Society and Development, v. 10, n. 8, e58710817657, 2021

(CC BY 4.0) | ISSN 2525-3409 | DOI: http://dx.doi.org/10.33448/rsd-v10i8.17657

Tao, J. et al.(2018). Elevation-dependent effects of climate change on vegetation greenness in the high mountains of southwest China during 1982-2013. Int. J. Climatol., 38, 2029-2038.

Tan, J. et al. (2014). A bibliometric analysis of research on proteomics in Science Citation Index Expanded. Scientometrics., 98, $1473-1490$.

Tai, T. C. \& James, W. R. (2018). Enhancing Climate Change Research With Open Science. Front. Environ. Sci., 6 (115), 1-5.

THE ENDNOTE TEAM. (2013). EndNote. Clarivate. Version X9. https://endnote.com/

Van Eck, N. J. \& Waltman, L. (2010). VOSViewer: Visualizing Scientific Landscapes [Software]. Available from https://www.vosviewer.com

Wang, X. et al. (2014). Alpine Cold Vegetation Response to Climate Change in the Western Nyainqentanglha Range in 1972-2009. The Scientific World Journal, 2014, 1-9.

Wang, L. et al. (2019). Bibliometric Analysis of Remote Sensing Research Trend in Crop Growth Monitoring: A Case Study in China. Remote Sens., $11,809$.

Walker, D. A. et al. (2005). The Circumpolar Arctic vegetation maJournal of Vegetation Science, 5 (16), 267-282.

Santin-Janin, H. et al. (2009). Assessing the performance of NDVI as a proxy for plant biomassusing non-linear models: a case study on the Kerguelenarchipelago. Polar Biol., 32, 861-871.

Shen, M. G. et al. (2015). Plant phenological responses to climate change on the Tibetan Plateau: research status and challenges. National Science Review, 2 (4), 454-467.

Sun, J. et al. (2006). Impact of climate change in the Hengduan Mountains of northwestern Yunnan, R. China: Vegetation distribution change in foretime and future. Proc. of SPIE, 6296, p.62960X-1.

Pouliot, D. et al. (2009). Trends in vegetation NDVI from 1 km AVHRR data over Canada for the period 1985-2006. International Journal of Remote Sensing, $30(1), 149-168$.

Jeong, S. J. et al. (2011). Phenology shifts at start vs. end of growing season in temperate vegetation over the Northern Hemisphere for the period $1982-2008$. Glob. Chang. Biol. 17, 2385-2399.

$\mathrm{Xi}, \mathrm{Z}$. (2020). China' s historical evolution of environmental protection along with the forty years' reform and opening-uEnvironmental Science and Ecotechnology, 1, 100001.

Zhang, R. et al. (2019a). Grassland vegetation phenological variations and responses to climate change in the Xinjiang region, China. Quaternary International, $513,56-65$.

Zhang, L. et al. (2016). A review of ecosystem services: a bibliometric analysis based on web of science. Acta Ecologica Sinica., 36, 5967-5977.

Zhang, X. et al. (2019b). Bibliometric analysis of highly cited articles on ecosystem services. PLoS ONE, 14 (2), e0210707.

Zhang, J. et al. (2016). Comparing keywords plus of WOS and author key-words: A case study of patient adherence research. J Assoc Inf Sci Technol., 67, 967972.

Zhang, J. et al. (2007). Evaluation of Grassland Dynamics in the Northern-Tibet Plateau of China Using Remote Sensing and Climate Data. Sensors, 7, 33123328.

Zhou, L. H. et al. (2009). Response of Vegetation Coverage on Climate Change in Arid Mountain of Northwest China. In: Ieee International Geoscience and Remote Sensing Symposium, Vols 1-5. Ieee: 1871. 